\title{
How to Measure Leaf Disease Damage Using Image Analysis in Image ${ }^{1}$
}

\author{
Lillian Pride, Gary Vallad, and Shinsuke Agehara²
}

This article introduces simple image processing and analysis techniques to quantify leaf disease damage using ImageJ, an open-source image processing program (Figure 1). These techniques are not meant to replace crop scouting or disease diagnosis by a plant diagnostic laboratory, but rather to provide a supplemental tool for making quantitative measurements of leaf disease damage. Similar techniques are also available for plant growth assessment, including plant height, plant width, and canopy cover area (Agehara 2020). The image processing and analysis techniques introduced in this article are fairly simple to use and thus can be adopted not only by researchers, but also by crop consultants, Extension agents, and students. Tutorial videos are also available at the UF IFAS Horticultural Crop Physiology Lab YouTube channel (https://www.youtube.com/pla ylist?list=PL4qrjj3jZ6i568ToiUV-DvAsQ0Gyb30hK).

\section{Introduction}

Plant diseases can cause many types of symptoms. Each disease has characteristic symptoms, such as leaf spots, chlorosis, necrosis, stunting, wilting, and other deformities. Although symptoms can be exhibited by the entire plant or by a specific organ of the plant, many common diseases cause symptoms on leaves.

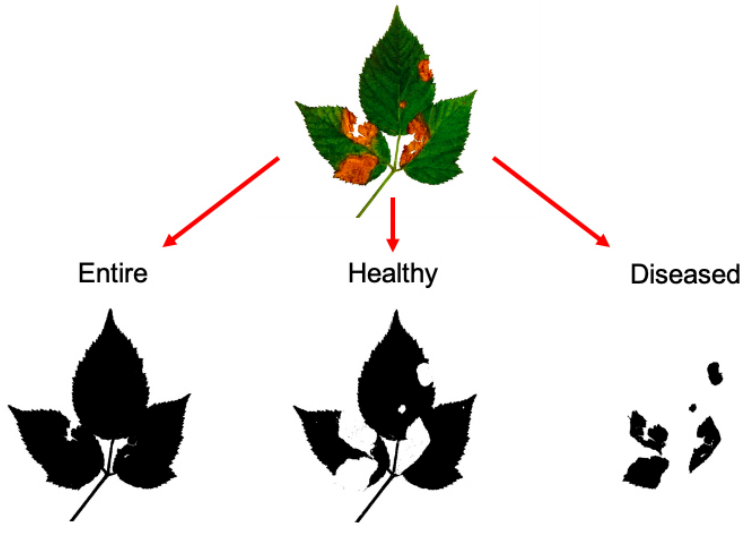

Figure 1. An example of image processing techniques for image-based quantification of leaf disease damage using ImageJ.

Image-based techniques can perform quantitative measurements that are generally more objective, accurate, and repeatable than visual scoring (Laflamme et al. 2016; Mutka and Bart 2015; Sibiya and Sumbwanyambe 2019; Xie et al. 2012). This article introduces simple image processing and analysis techniques to quantify leaf disease damage using ImageJ, an open-source image processing program. In the following sections, we will demonstrate different techniques step by step using the following plant diseases as examples: Phomopsis leaf blight on blackberry, downy mildew on cucumber, and bacterial leaf spot on tomato. We will also demonstrate some troubleshooting techniques to process nonideal images. The advantages and disadvantages of

1. This document is HS1382, one of a series of the Horticultural Sciences Department, UF/IFAS Extension. Original publication date September 2020. Visit the EDIS website at https://edis.ifas.ufl.edu for the currently supported version of this publication.

2. Lillian Pride, research technician; Gary Vallad, associate professor, Plant Pathology Department; and Shinsuke Agehara, assistant professor, Horticultural Sciences Department, UF/IFAS Gulf Coast Research and Education Center, Wimauma, FL 33598.

The use of trade names in this publication is solely for the purpose of providing specific information. UF/IFAS does not guarantee or warranty the products named, and references to them in this publication does not signify our approval to the exclusion of other products of suitable composition.

The Institute of Food and Agricultural Sciences (IFAS) is an Equal Opportunity Institution authorized to provide research, educational information and other services

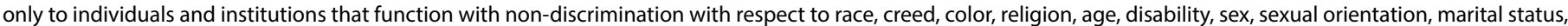

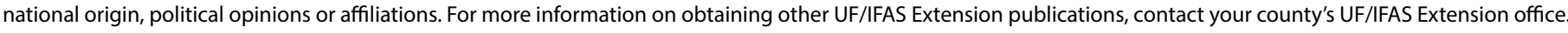
U.S. Department of Agriculture, UF/IFAS Extension Service, University of Florida, IFAS, Florida A \& M University Cooperative Extension Program, and Boards of County Commissioners Cooperating. Nick T. Place, dean for UF/IFAS Extension. 
image-based quantification of leaf disease damage are as follows:

\section{Advantages}

- Image-based measurements provide quantitative data and can be more objective and accurate than visual scoring.

- Cost and tools needed are minimal-you only need an image-capturing device (digital camera, smartphone, or flatbed scanner) and ImageJ.

- Multiple measurements can be performed on the same image (e.g., disease infection area, percentage of disease infection, and leaf spot counts).

- Images themselves are very good visual data.

- Photographs can be saved with metadata, such as geolocation, date, and time, automatically.

\section{Disadvantages}

- Image-based measurements cannot distinguish between different diseases and symptoms caused by different factors (e.g., disease vs. abiotic stress).

- Accuracy of measurements can be affected by the image quality and the severity of disease damage.

- Using ImageJ for image processing and analysis requires some basic computer literacy and the installation of a compatible version of Java software (https://www.java. com/ES/download/).

\section{How to Capture Ideal Images for Image Analysis}

Obtaining high-quality images is critical to both qualitative interpretations and quantitative measurements via image analysis. High-quality images can also save a significant amount of time in image processing. Here are some tips to take ideal images for image analysis:

- Use a flatbed scanner instead of a camera. Scanned images have several advantages over photographs, including better color depth, uniform lighting, no optical distortion, and minimized leaf curvature effects. We recommend using a resolution of 300 dots per inch (dpi) or higher.

- If taking photographs indoors, use a tripod and lay leaves on a high-contrast background (e.g., white piece of paper, black sheet). Include a reference object of known size in the image, so the image can be scaled.

- If taking photographs outdoors, choose an overcast day, or a camera angle that can minimize deep shadows in the image (e.g., a sunny day with no clouds at 12-2 pm for overhead plot photographs).

\section{Download ImageJ Software}

The tutorial video for this step is available at https://youtu. be/KMq4cV_BQ7Y.

1.Go to https://imagej.nih.gov/ij/ and select "Download."

2. Determine your platform (Mac/Linux/Windows) and click on the appropriate link.

3. Install the program. The measurements described below do not require plugins.

Note:

- Another download option is Fiji (https://imagej.net/ Fiji/Downloads), a version of ImageJ bundled with many plugins.

- There are also other useful plugins available for separate download (e.g., Wika Segmentation for training ImageJ to automatically process images).

- The version of ImageJ used in this article is 1.52 .

\section{Set Scale (Image Size Calibration) Photographs}

The tutorial video for this step is available at https://youtu. be/HoidaLxKMMk.

1.Import the image: Go to "File > Open" and navigate to the image or drag the file onto the ImageJ toolbar. Below is a photograph of blackberry leaves laid flat on a piece of white copy paper. The photograph was taken using a compact digital camera (SONY DSC-RX100) in a room with only ceiling lights.

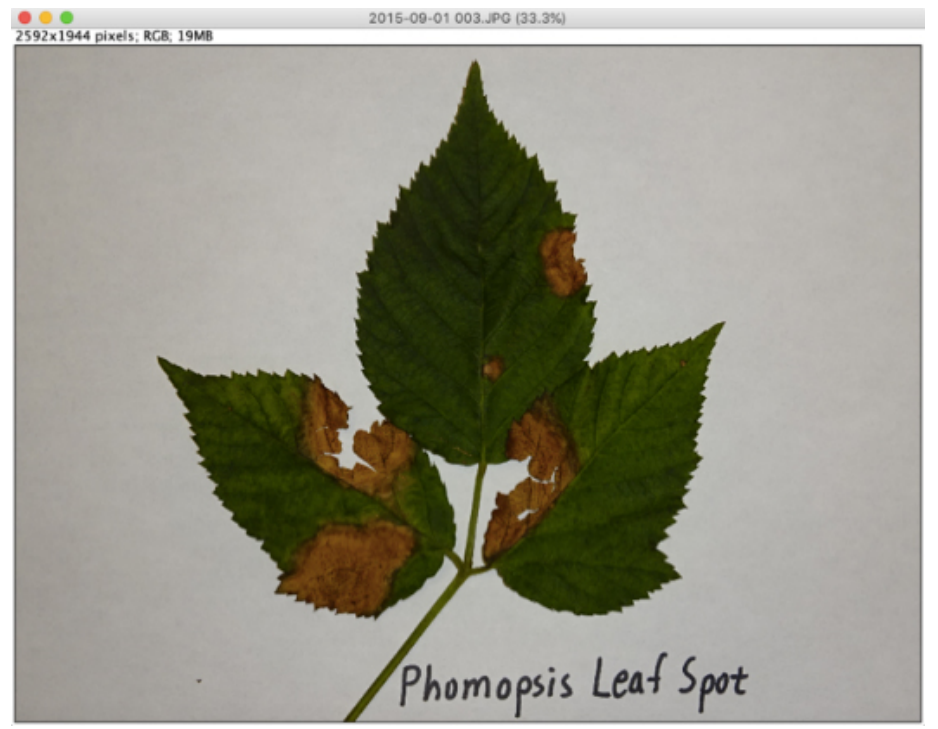


2. Select the "Straight Line" tool (highlighted below).

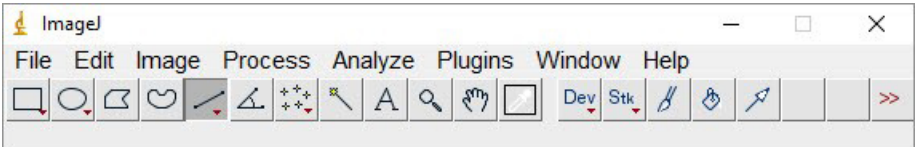

3. Draw a line to an object of known size (e.g., ruler).

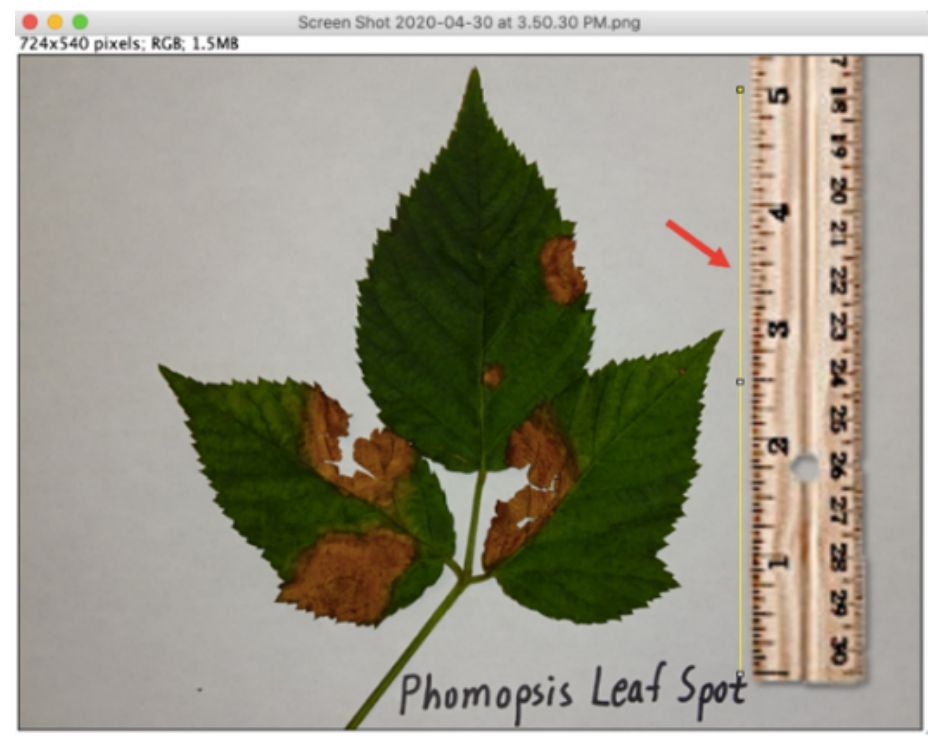

4. Go to "Analyze > Set Scale" to set the distance to the line.

i) Enter the following parameters (e.g., ruler height $=5$ inches).

- Known distance: 5

- Unit of length: inch

- Global: Checked

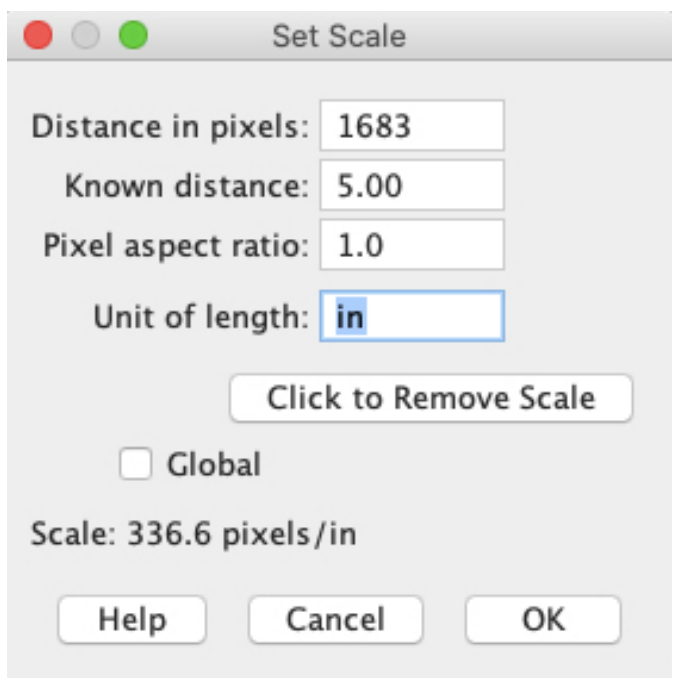

ii) Click on "OK."
Note:

- For accurate image size calibration, the reference object should be large and near the object of interest.

- When the "Global" option is checked, the same image calibration will be applied to images that will be imported subsequently. Use this option when the same image needs to be imported multiple times to perform different sets of image analysis or when all images in a set have the same scale (e.g., scanned images, images taken using a tripod). When working on an image with a different scale, "Set Scale" must be done again.

\section{Scanned Images}

The tutorial video for this step is available at https://youtu. be/9vnIt0JXJ5M.

1. Obtain the pixel dimensions, resolution, and physical size of the image.

Note:

- On Windows, the image's pixel dimensions and resolution in dots per inch (dpi) can be obtained by right-clicking the file, opening "Properties," and then viewing the "Details" tab. To calculate the physical image size, divide the pixel dimensions by the resolution.

- On Mac, the image's physical size in inches, dpi, and pixel dimensions can be obtained by opening the file in "Preview" and going to "Tools > Adjust Size...."

2. Import the image in Image J. Below is a scanned image in $3400 \times 4680$ pixels at $400 \mathrm{dpi}(8.5 \times 11.7$ inches $)$ of a tomato leaf obtained using a flatbed scanner (Epson Perfection V800).

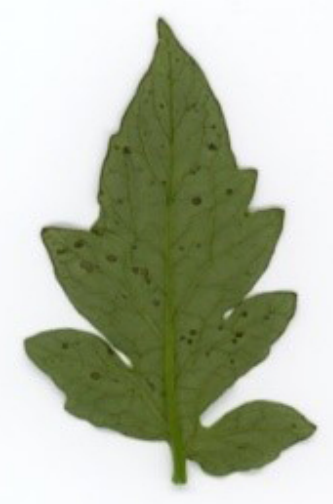

3. Go to "Analyze > Set Scale" to calibrate the image.

i) Enter the following parameters.

- Distance in pixels: 3400 
- Known distance: 8.5

- Unit of length: inch

- Global: Checked

ii) Click on "OK."

Note:

- In ImageJ, pixel dimensions are listed on the left upper corner of the image window.

\section{Area Measurement}

\section{Example 1: Blackberry Phomopsis Leaf Blight (Sampled Leaves)}

The tutorial video for this step is available at https://youtu. be/tvosUZh6oDI.

This example will use the photograph of the blackberry leaves used in the "Set Scale" section. Phompsis leaf blight is displayed as large necrotic lesions.

1. Go to "Image $>$ Adjust $>$ Color Threshold" to separate the leaf from the background.

i) Adjust color threshold parameters (hue, saturation, and brightness) to select the entire leaf (healthy + diseased).

\section{Note:}

- Hue is one of the color appearance parameters, and it describes pure spectrum colors. For the entire leaf area, the hue range from 0 to 110 works well for most images.

- The optimum values can vary depending on the image quality, particularly for saturation and brightness.

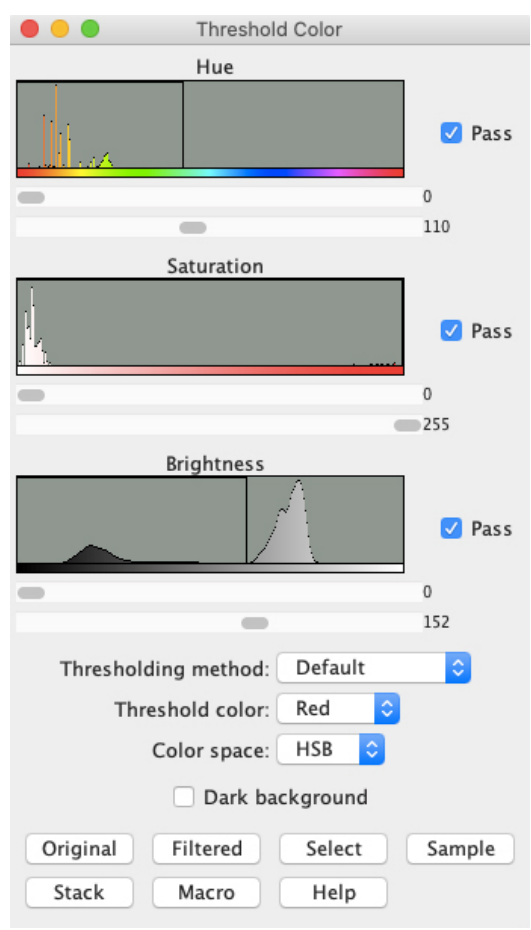

ii) Click on "Select."

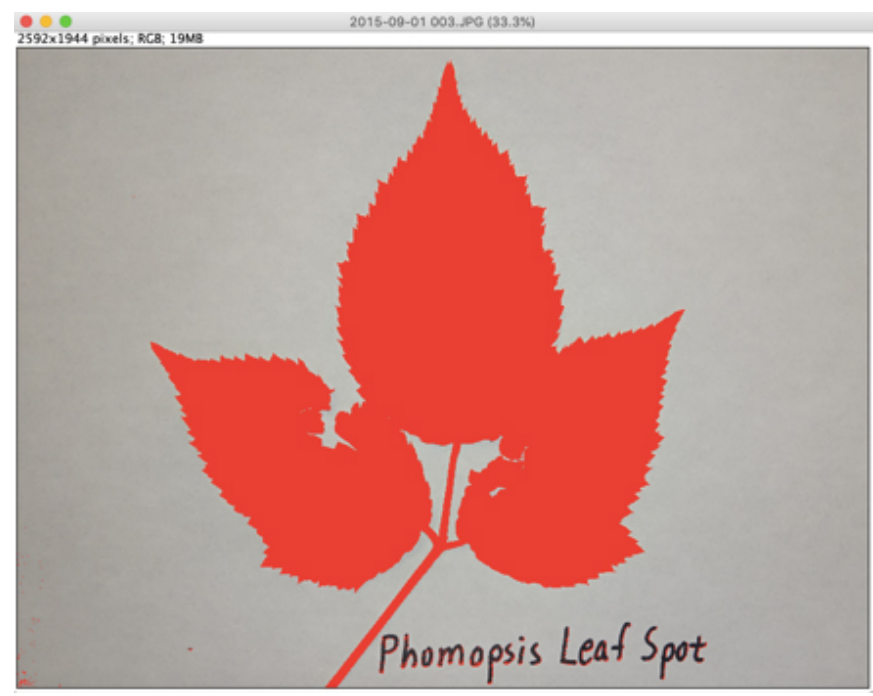

2. Go to "Process $>$ Binary $>$ Make Binary" to convert the color image to a binary (black and white) image. 


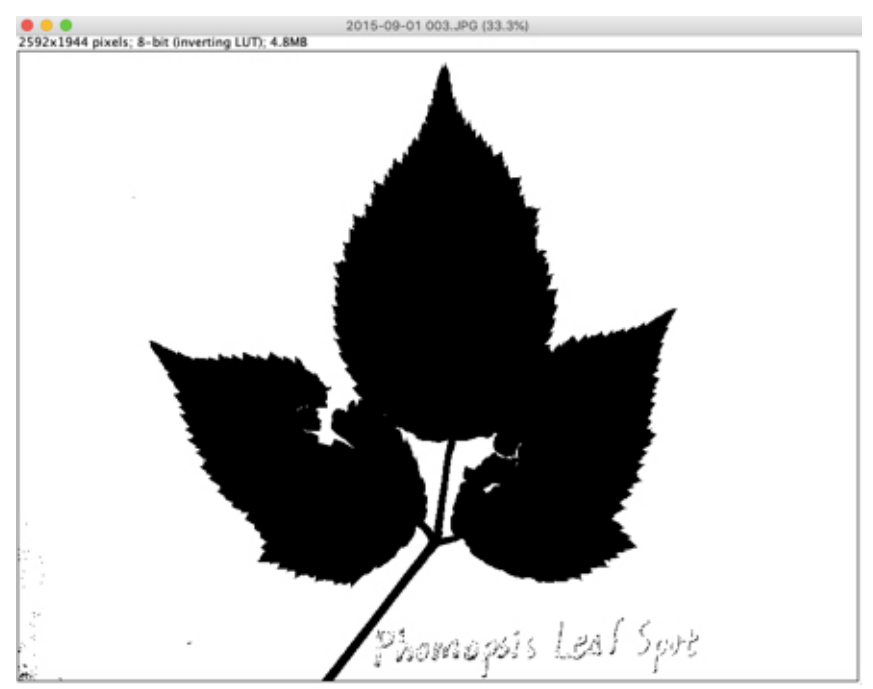

3. Use the "Paintbrush Tool" to remove large clusters of pixels that are not part of the leaves.

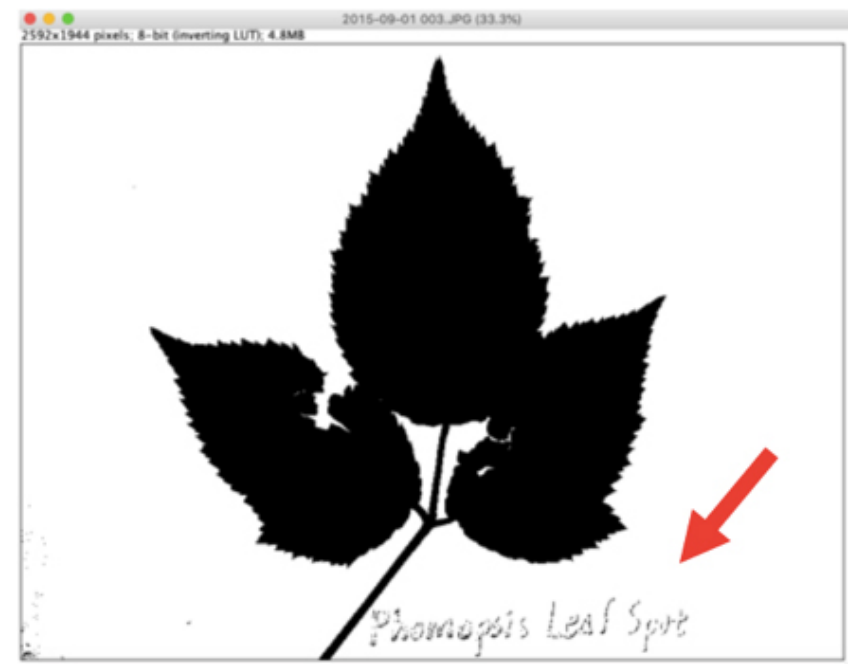

i) Double click the "Paintbrush Tool" (highlighted below).

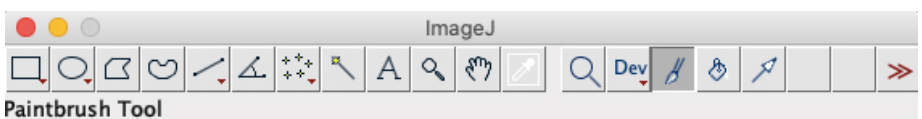

Paintbrush Too

ii) Select the following parameters.

- Brush width: 50

- Color: White

- Paint on overlay: Unchecked

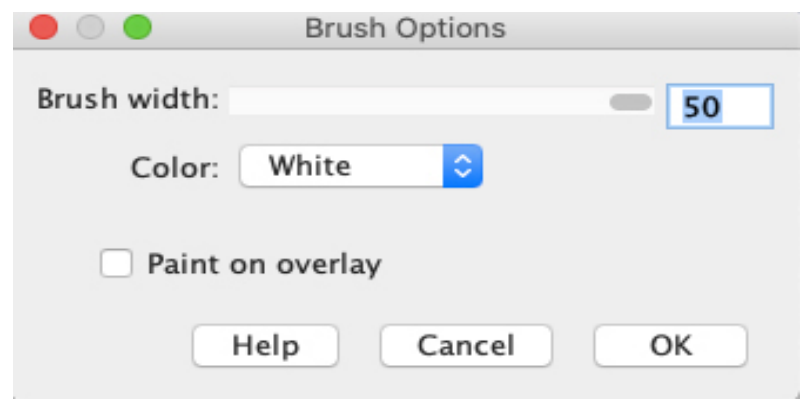

iii) Click on "OK" and erase the pixels that are not part of the leaves.

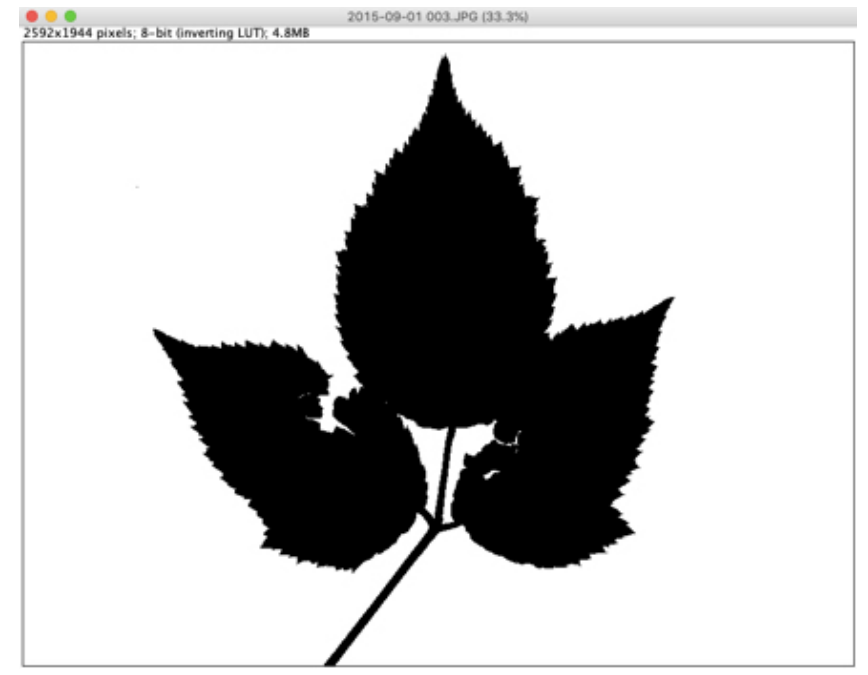

4. Go to "Process $>$ Noise $>$ Remove Outliers" to remove small specks that are not part of the leaves. This step can be skipped when the image has a clean background as in this example.

5. Select measurement variables and perform the measurement of the entire leaf area.

i) Go to "Analyze > Set Measurements" and select "Area" and "Limit to threshold."

ii) Click on "OK."

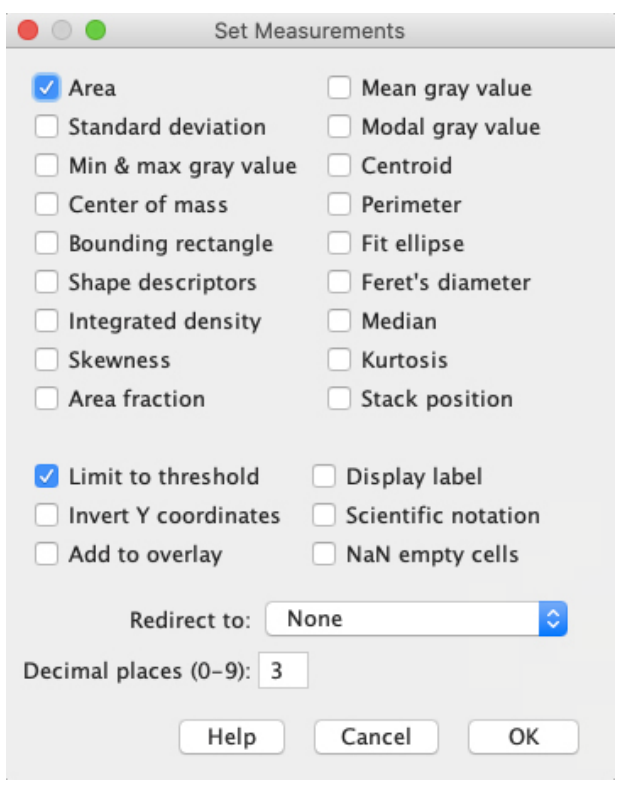

iii) Go to "Analyze $>$ Measure" or Ctrl+M to measure the entire leaf area.

6. Reopen the same image and go to "Image $>$ Adjust $>$ Color Threshold" to select the diseased leaf area. 
i) Adjust color threshold parameters (hue, saturation, and brightness).

Note:

- The hue range from 0 to 40 works well to select the diseased leaf area in this image.

- The optimum hue range varies depending on the severity of chlorosis or necrosis and the greenness of the healthy leaf area.

ii) Click on "Select."

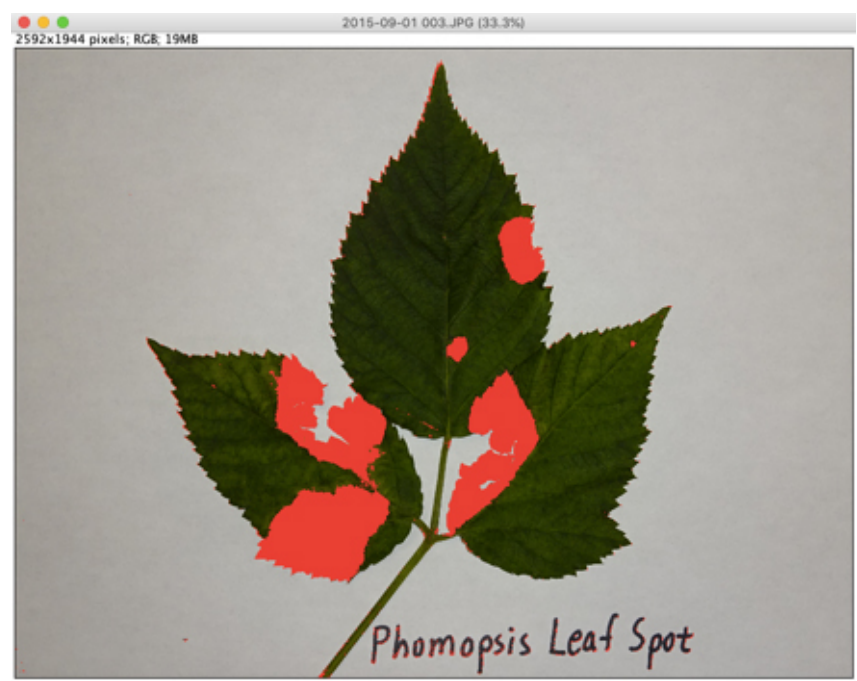

7. Go to "Process $>$ Binary $>$ Make Binary" to convert the color image to a binary image.

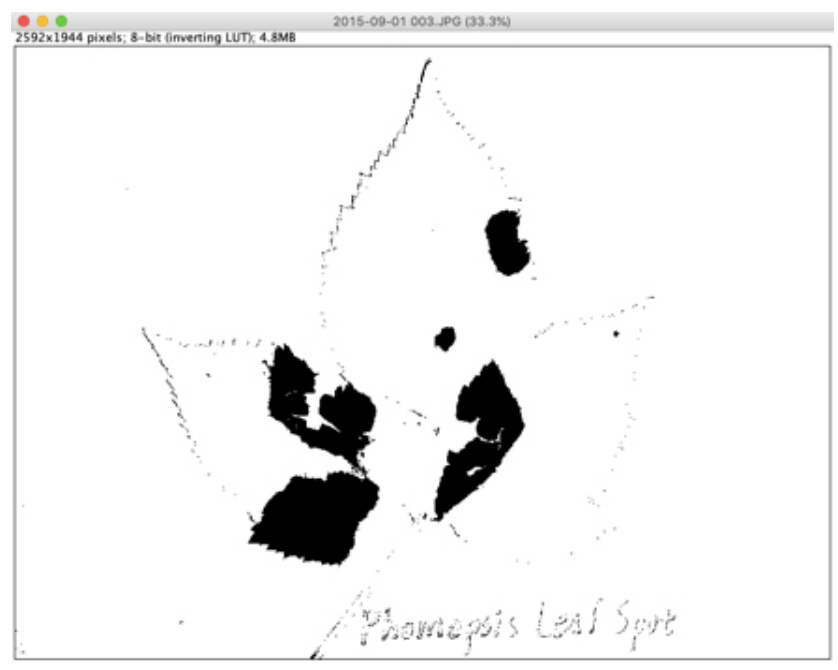

8. Use the "Paintbrush Tool" to remove large clusters of pixels that are not necrotic lesions.

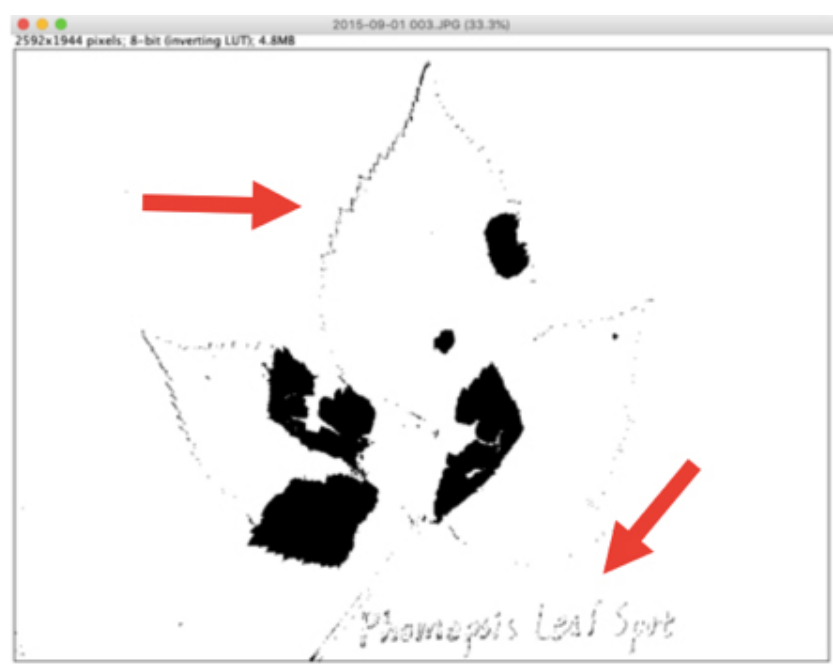

9. Go to "Process $>$ Noise $>$ Remove Outliers" to remove small specks that are not necrotic lesions.

i) Select the threshold as follows.

- Radius: 5

- Threshold: 50

- Which outliers: Dark

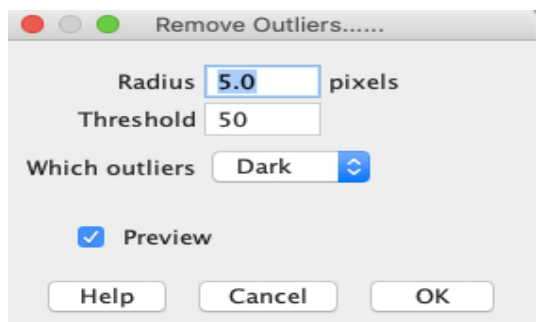

ii) Check on "Preview." If everything looks good, click on "OK."

Note:

- Increase the radius if outliers are not removed.

- Select the radius that can remove outliers without deleting a significant portion of lesions.

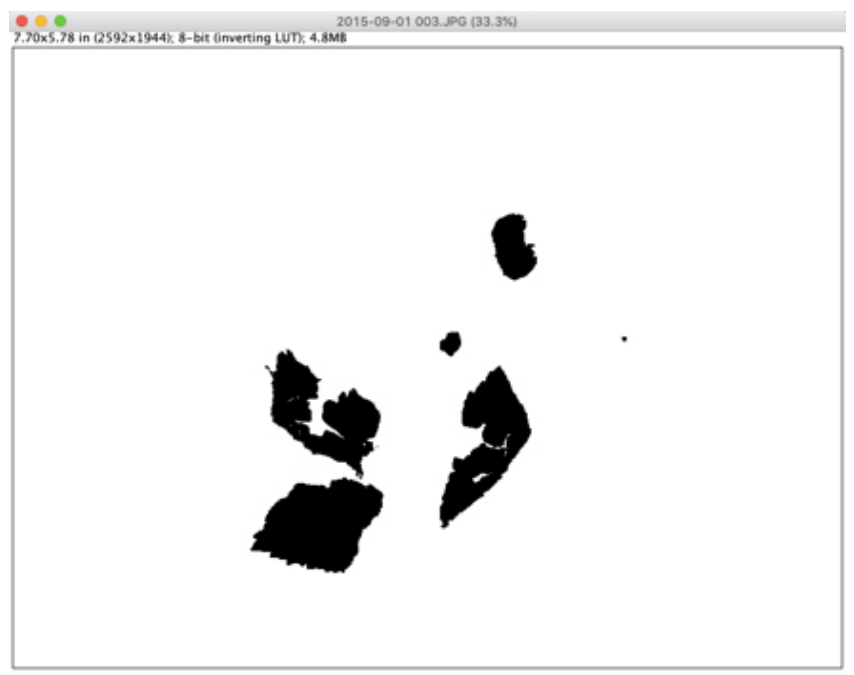


10. Go to "Analyze > Measure" or Ctrl $+\mathrm{M}$ to measure the diseased leaf area.

11. Calculate the percentage of disease damage as follows: Diseased leaf area $\div$ Entire leaf area $\times 100$. Figure 1 shows blackberry leaf images processed by ImageJ to measure the entire, healthy, and diseased leaf areas.

\section{Example 2: Cucumber Downy Mildew (Multiple Plants)}

The tutorial video for this step is available at https://youtu. be/elyYvOEUezM.

This example will use a photograph of cucumber plants in the field. The photograph was taken using a compact digital camera (SONY DSC-RX100) in the middle of a sunny day. Damage caused by downy mildew is displayed as necrosis on the vines.

1. Import the image.

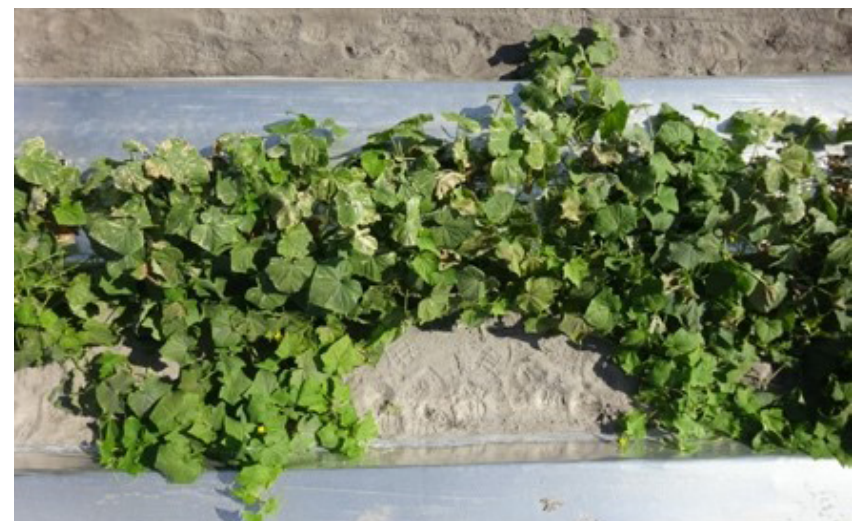

2. Go to "Image > Adjust > Color Threshold" to separate the canopy from the background.

i) Adjust color threshold parameters (hue, saturation, and brightness) to select the entire canopy (healthy + diseased).

Note:

- In this image, hue between 0 and 122, saturation between 58 and 255, and brightness between 9 and 255 work well. ii) Click on "Select."

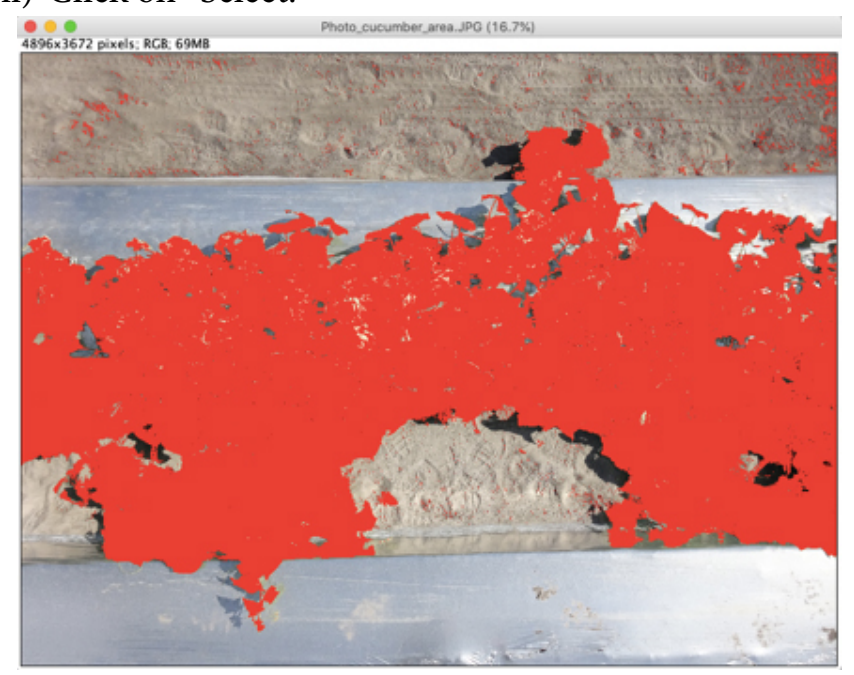

3. Go to "Image > Color > Color Picker" to set the background color to white.

i) Double-click the "B" in the $\mathrm{CP}$ window.

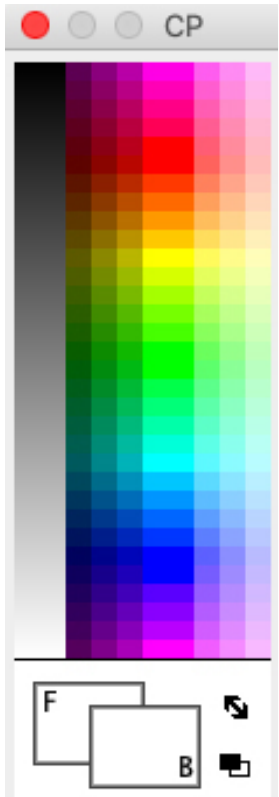

ii) The Background Color window opens. Fill 255 in all three cells in the Background Color window.

iii) Click "OK" to close the Background Color window.

iv) Close the CP window.

Note:

- Now if the background is cleared, it will be white instead of black. 
4. Go to "Edit > Clear Outside" to remove everything but the selected area.

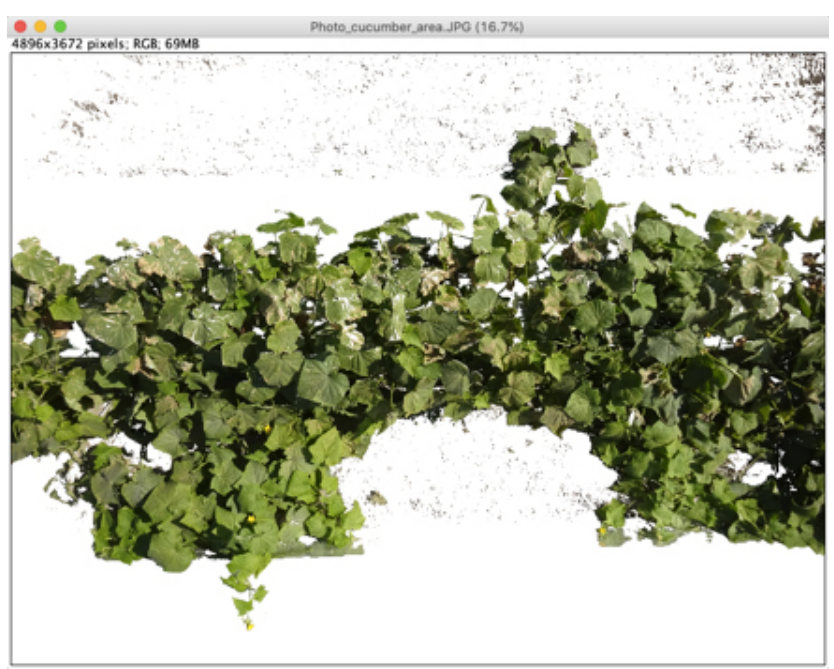

5. Use the "Paintbrush Tool" or "Process $>$ Noise $>$ Remove Outliers" to remove pixels that are not leaves.

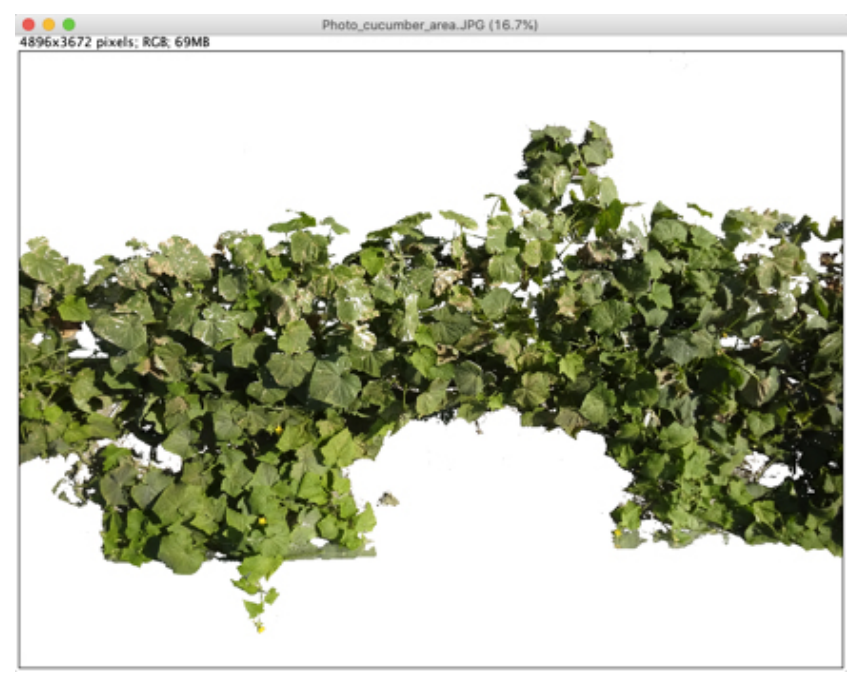

6. Go to "File $>$ Save As... > Tiff..." to save the image as a TIFF file. The saved image will be used in Steps 8-10 to measure the entire canopy area and in Steps 12-14 to measure the diseased canopy area.

7. Close the image window and open the image saved in Step 6.
8. Repeat Step 2 to select the canopy.

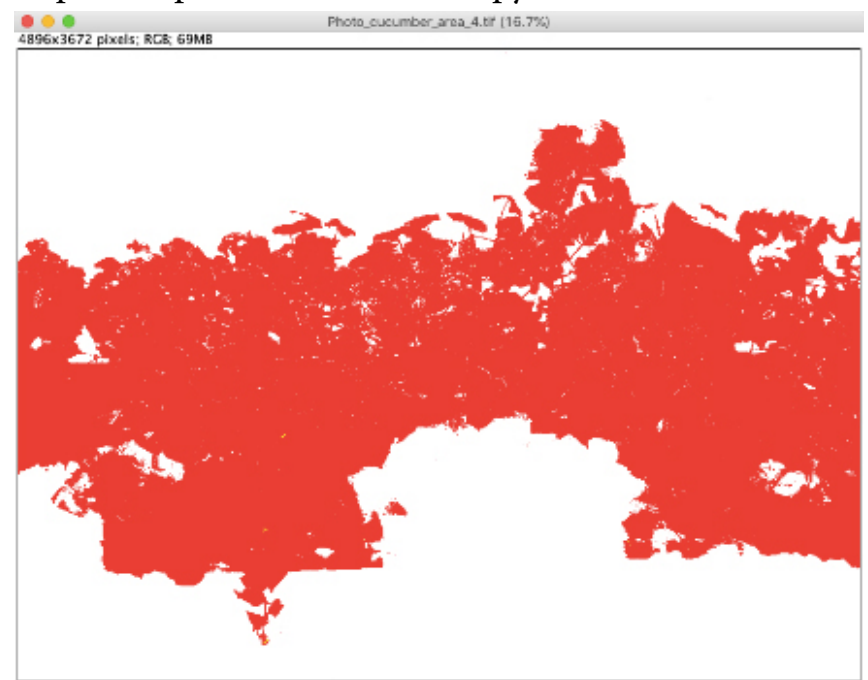

9. Go to "Process > Binary > Make Binary" to convert the color image to a binary image.

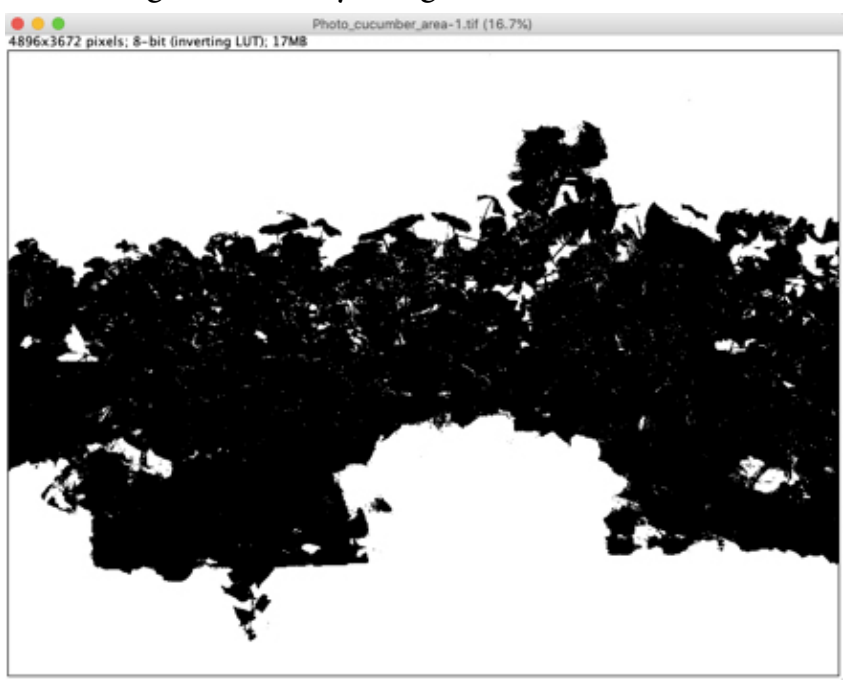

10. Go to "Analyze > Measure" or Ctrl $+\mathrm{M}$ to measure the entire canopy area.

11. Close the image window and reopen the image saved in Step 6.

12. Go to "Image > Adjust > Color Threshold" to select the diseased canopy area.

i) Adjust color threshold parameters (hue, saturation, and brightness).

Note:

- In this image, hue between 0 and 45 , saturation between 0 and 155, and brightness between 0 and 254 work well.

ii) Click on "Select." 


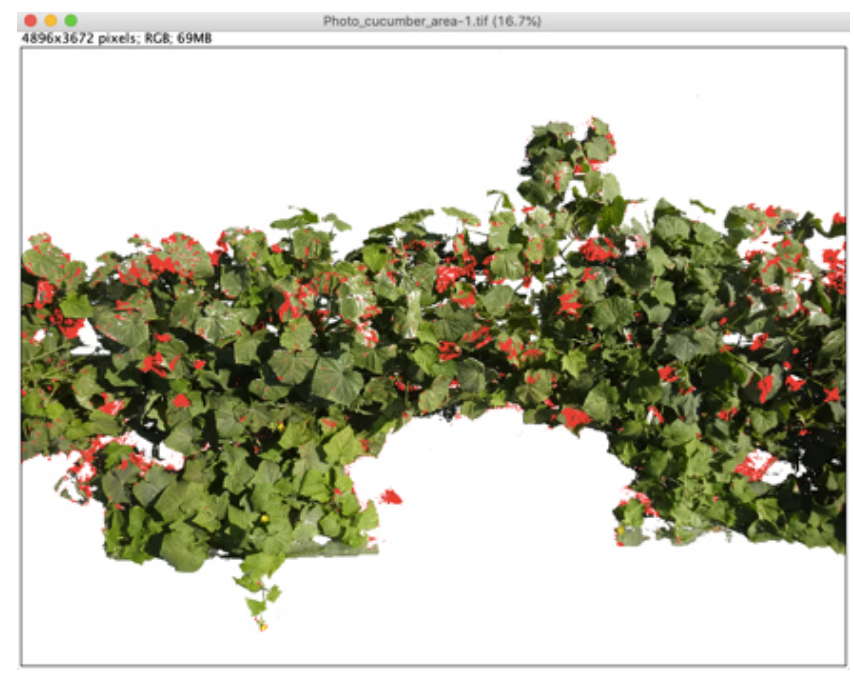

13. Go to "Process $>$ Binary $>$ Make Binary" to convert the color image to a binary image.

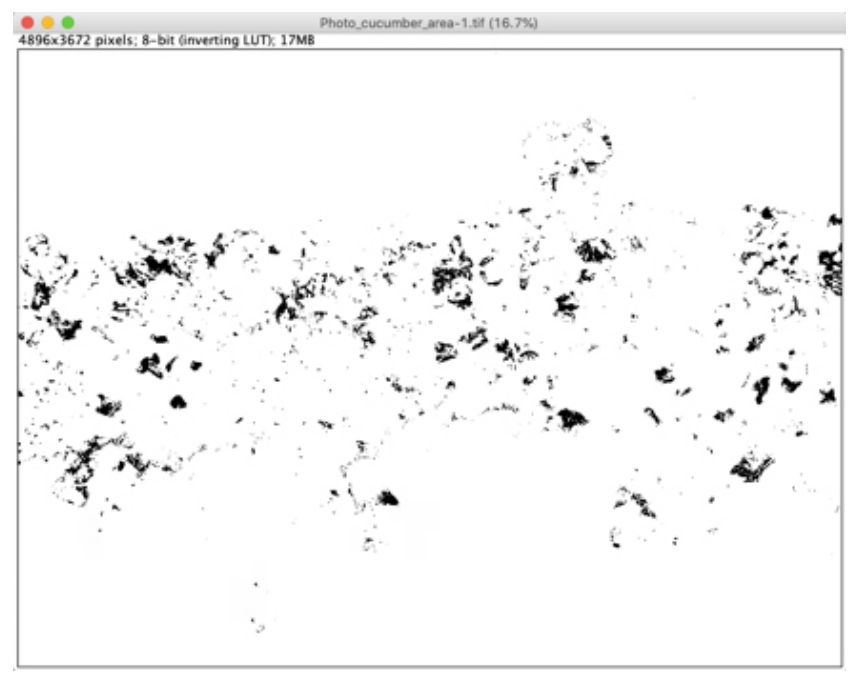

14. Go to "Analyze > Measure" or Ctrl $+\mathrm{M}$ to measure the diseased canopy area.

15. Calculate the percentage of disease damage as follows:

Diseased canopy area $\div$ Entire canopy area $\times 100$.

\section{Count Measurement (Tomato}

\section{Bacterial Leaf Spot)}

\section{Example 1: Scanned Image}

The tutorial video for this step is available at https://youtu. be/YV5L_YDMkRo.

This example will use the tomato leaf scan used in the "Set Scale $>$ Scanned Images" section. Tomato bacterial leaf spot is displayed as small dots on the back of the leaf.
1. Click the "Rectangle" tool (highlighted below).

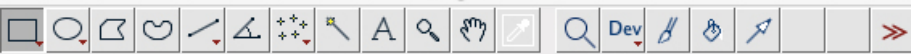
*Rectangle*, rounded rect or rotated rect (right click to switch)

2. Select the smallest rectangle possible around the leaf.

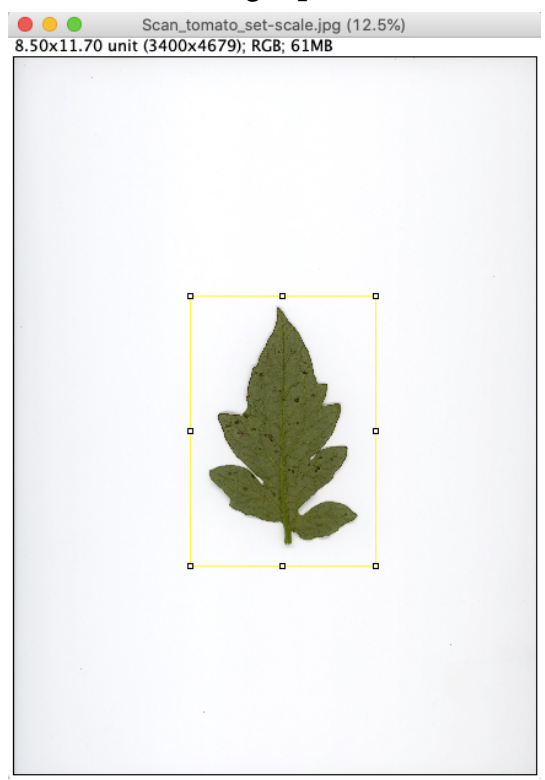

3. Go to "Image > Crop."

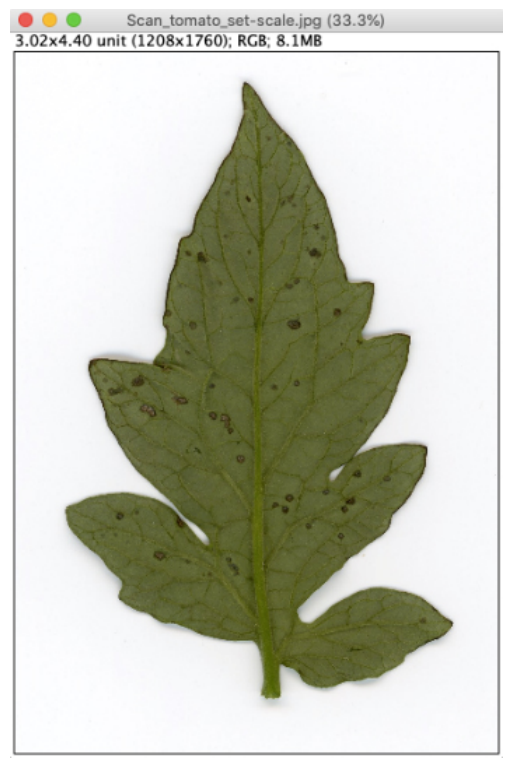

4. Go to "Image > Adjust > Color Threshold" to select leaf spots.

i) Adjust color threshold parameters (hue, saturation, and brightness).

Note:

- In this image, hue between 0 and 47 , saturation between 0 and 255, and brightness between 0 and 147 work well.

ii) Click on "Select." 


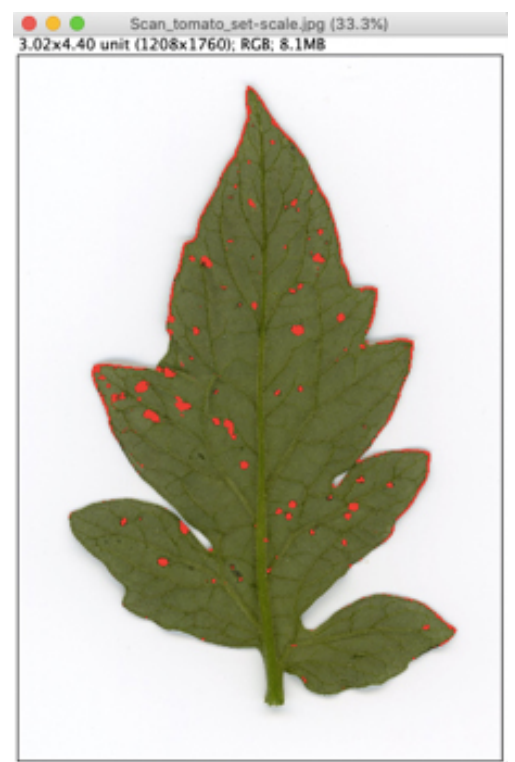

5. Go to "Process $>$ Binary $>$ Make Binary" to convert the color image to a binary image.

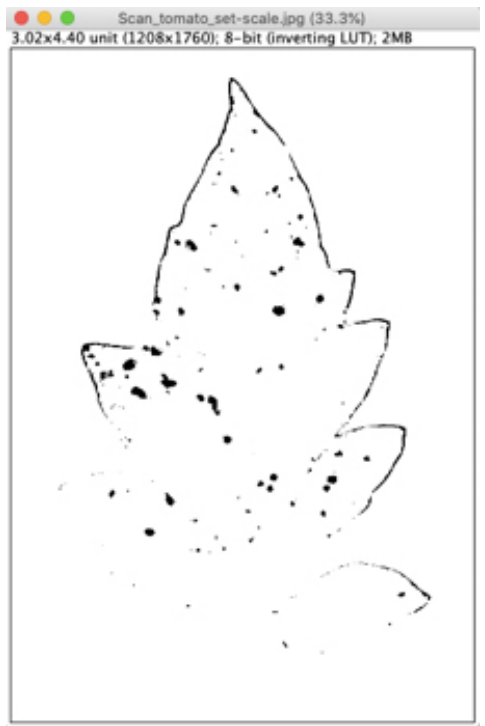

6. Use the "Paintbrush Tool" to remove large clusters of pixels that are not leaf spots.

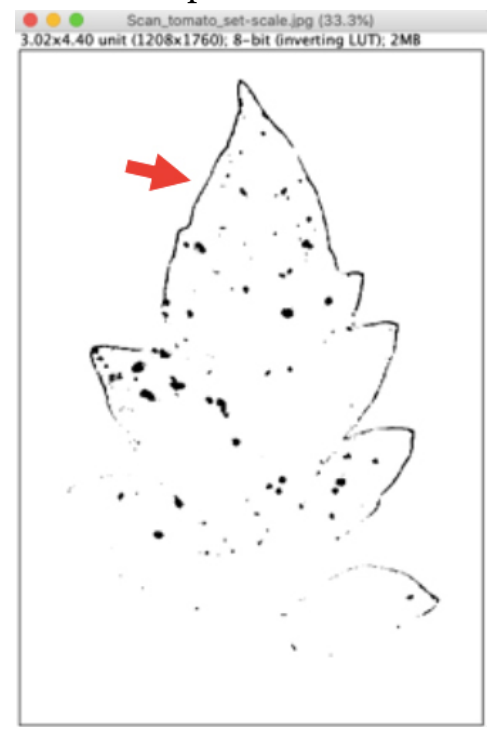

7. Go to "Process $>$ Noise $>$ Remove Outliers" to remove small specks that are not leaf spots.

i) Select the threshold as follows.

- Radius: 1

- Threshold: 50

- Which outliers: Dark

ii) Check on "Preview." If everything looks good, click on "OK."

Note:

- To avoid deleting leaf spots in this step, open the original image and compare it to the edited image.

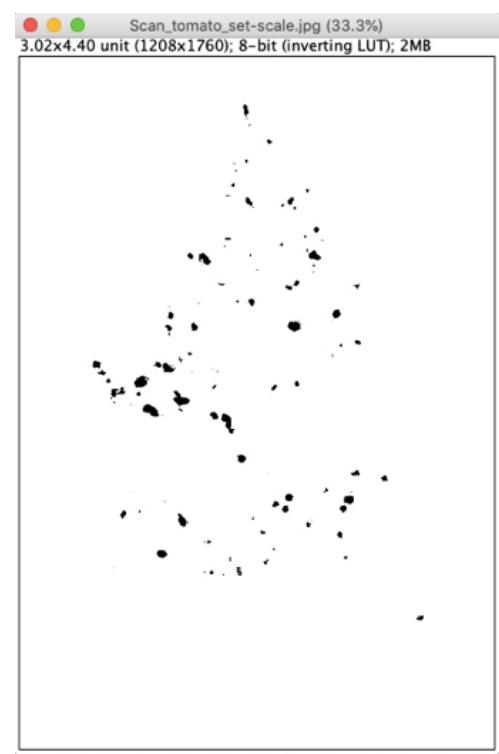

8. Go to "Analyze > Analyze Particles" to measure the number of leaf spots.

i) Enter the following parameters.

- Size (unit^^2): 0-Infinity.

- Circularity: 0.00-1.00

- Pixel units: Checked

- Show: Outlines

Note:

- Use the "Size" option to exclude pixels that are not leaf spots by size by setting the minimum and maximum pixel areas. In Steps 6-7, we removed all large and small particles that were not leaf spots. We can therefore count all remaining particles.

- Use the "Circularity" option to exclude pixels that are not leaf spots by roundness by setting the minimum and maximum circularity. Zero is not round at all and 1 is a perfect circle. 
- The "Show" option provides multiple possible visual outputs.

ii) Select the options checked in the image below.

iii) Click on "OK."

\begin{tabular}{|c|c|c|c|}
\hline 000 & \multicolumn{3}{|c|}{ Analyze Particles } \\
\hline Size (pixel^2): & \multicolumn{2}{|c|}{0 -Infinity } & \\
\hline Circularity: & \multicolumn{2}{|c|}{$0.00-1.00$} & \\
\hline Show: & Out & nes & $\hat{v}$ \\
\hline \multicolumn{2}{|c|}{$\checkmark$ Display results } & \multicolumn{2}{|c|}{$\checkmark$ Exclude on edges } \\
\hline \multicolumn{2}{|c|}{$\checkmark$ Clear results } & \multicolumn{2}{|c|}{$\checkmark$ Include holes } \\
\hline \multicolumn{2}{|l|}{$\checkmark$ Summarize } & \multicolumn{2}{|c|}{ Record starts } \\
\hline \multicolumn{2}{|c|}{ Add to Manager } & \multicolumn{2}{|c|}{ In situ Show } \\
\hline Help & & Cancel & OK \\
\hline
\end{tabular}

9. Record the desired information from the "Summary" and "Results" tables. The count data are indicated by a red arrow in the image below.

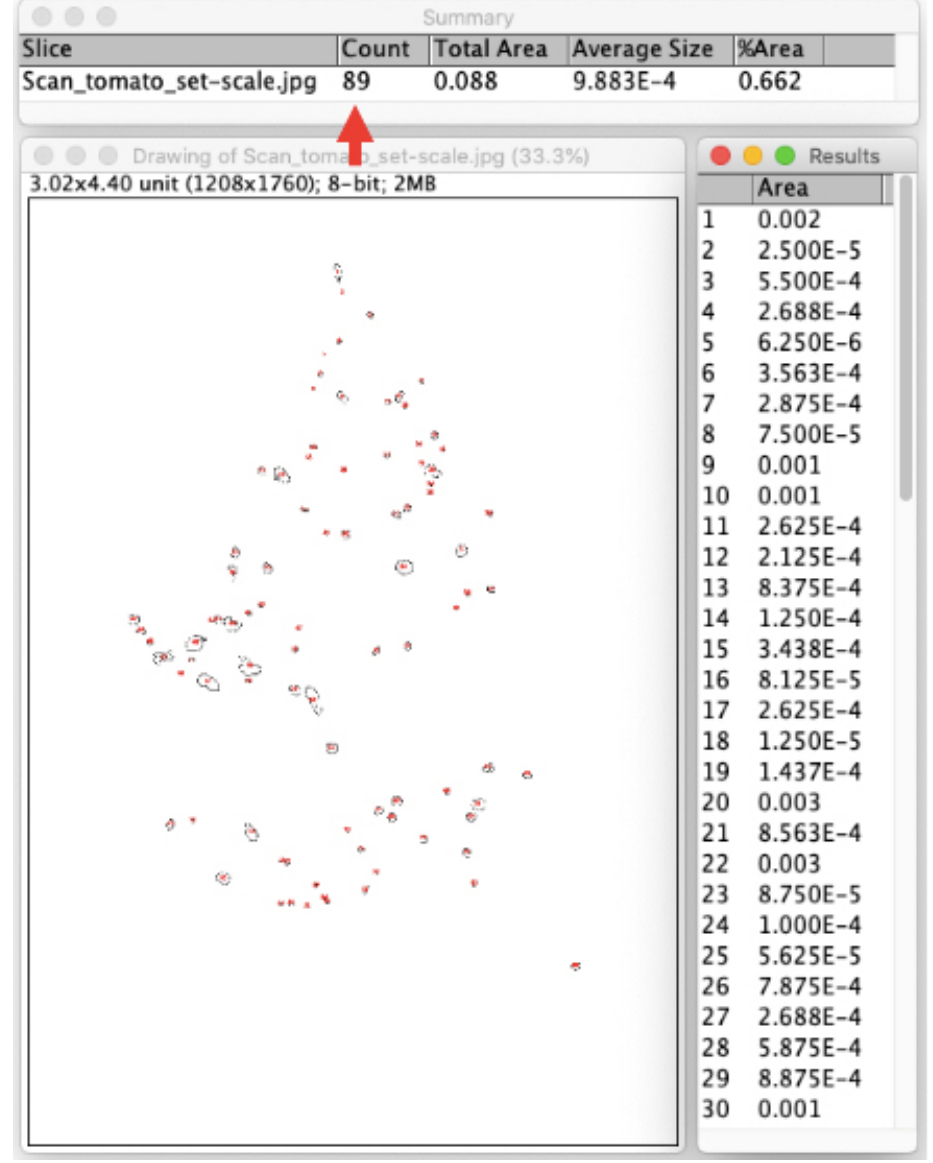

\section{Example 2: Field Photograph}

The tutorial video for this step is available at https://youtu. be/bDuj4J11OS8.

In this example, we will analyze a photograph of the same leaf from Example 1, taken in the field with a smartphone (iPhone 7). Because leaf curling has rendered some spots invisible and outdoor lighting has decreased the color contrast between the healthy leaf tissue and the diseased tissue, ImageJ will detect fewer leaf spots in the photograph than in the scanned image. However, it is still possible to count leaf spots using the following procedure.

1. Import the image.

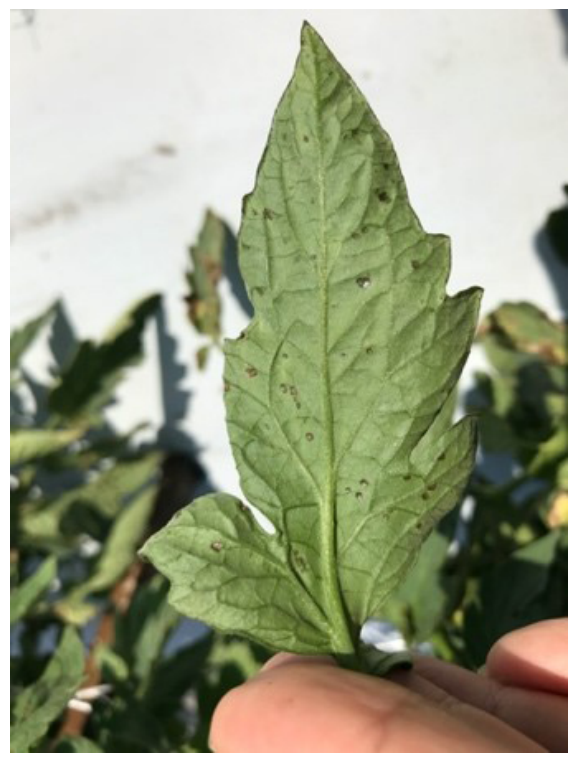

2. Use the "Freehand selections" tool to select the area of interest.

i) Click the "Freehand selections" tool (highlighted below).

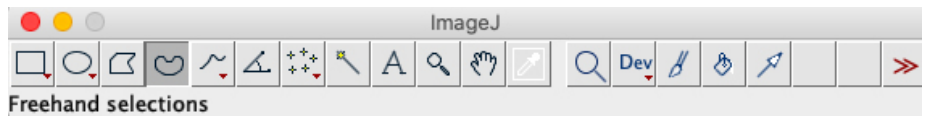

ii) Left-click and hold to start outlining.

iii) While continuing to hold, outline the entire desired area. 


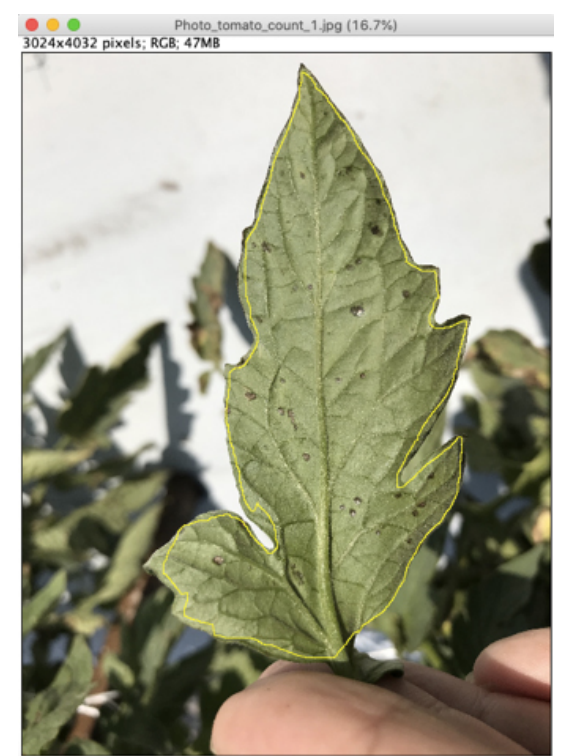

Note:

- For most consistent results, it may be necessary to standardize what part of the leaf to select. In this image, diseased tissues on the border of the leaf have been excluded.

3. Go to "Edit > Clear Outside" to remove the background.

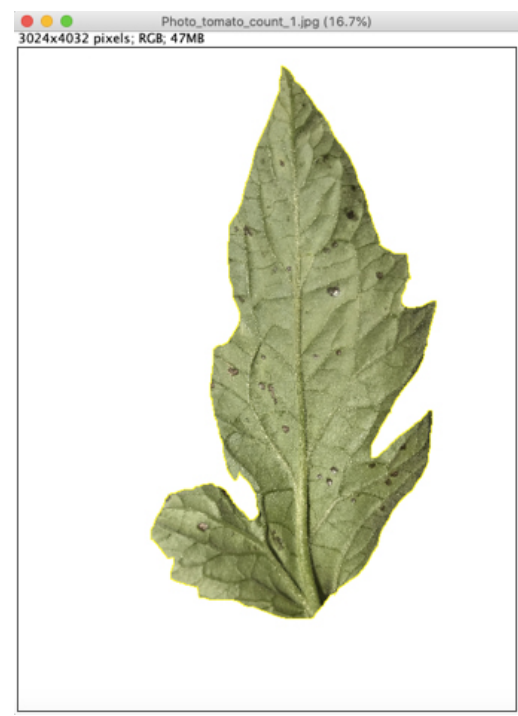

4. Go to "Image > Adjust $>$ Color Threshold" to select leaf spots.

i) Adjust color threshold parameters (hue, saturation, and brightness).

Note:

- In this image, hue between 0 and 47, saturation between 0 and 255, and brightness between 0 and 254 work well.

ii) Click on "Select."

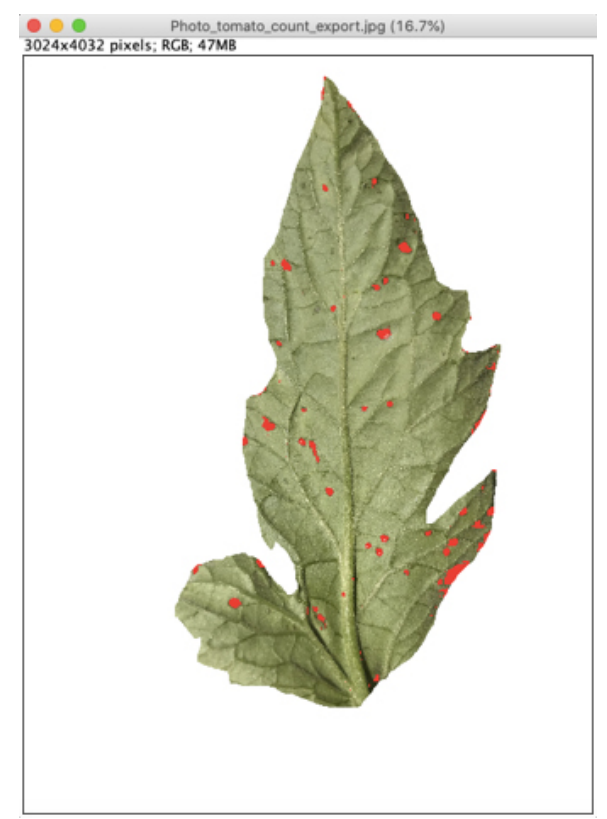

5. Go to "Process $>$ Binary > Make Binary" to convert the color image to a binary image.

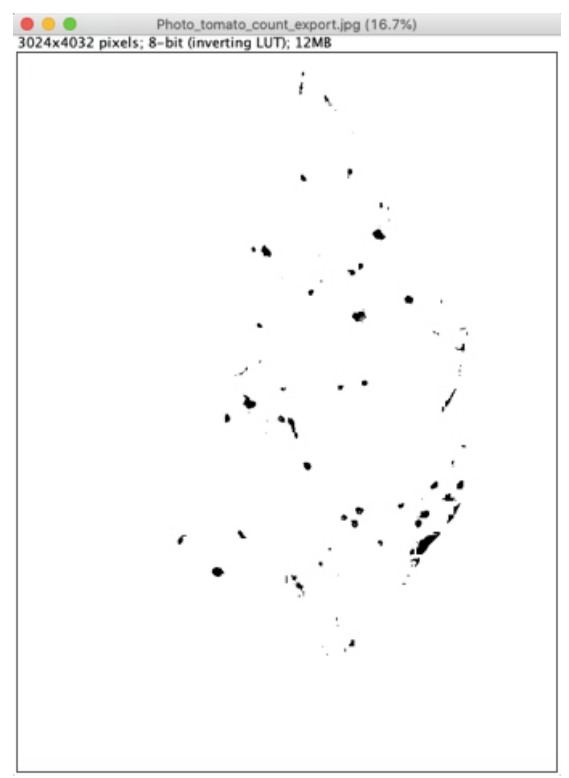

6. Use the "Paintbrush Tool" to remove large clusters of pixels that are not leaf spots.

7. Go to "Process $>$ Noise $>$ Remove Outliers" to remove small specks that are not leaf spots.

i) Select the threshold as follows.

- Radius: 2

- Threshold: 50

- Which outliers: Dark

ii) Check on "Preview." If everything looks good, click on "OK." 
8. Go to "Analyze > Analyze Particles" to measure the number of leaf spots.

i) Enter the following parameters.

- Size (unit^2): 0-Infinity.

- Circularity: 0.00-1.00

- Pixel units: Checked

- Show: Outlines

ii) Check the same options selected in the same step for Example 1.

iii) Click on "OK."

9. Record the desired information from the "Summary" and "Results" tables. The count data are indicated by a red arrow in the image below.

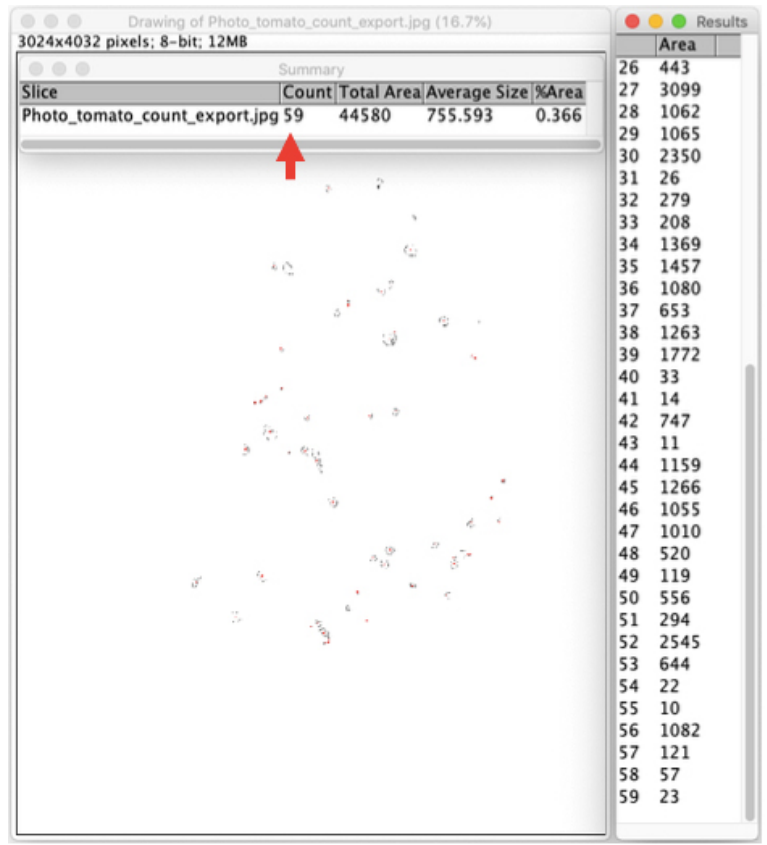

\section{Literature Cited}

Agehara, S. 2020. Simple Imaging Techniques for Plant Growth Assessment. HS1353. Gainesville: University of Florida Institute of Food and Agricultural Sciences. https:// edis.ifas.ufl.edu/hs1353.

Laflamme, B., M. Middleton, T. Lo, D. Desveaux, and D. S. Guttman. 2016. "Image-Based Quantification of Plant Immunity and Disease." Molecular Plant-Microbe Interactions 29:919-924.

Mutka, A. M., and R. S. Bart. 2015. "Image-Based Phenotyping of Plant Disease Symptoms." Frontiers in Plant Science 5:1-8.
Sibiya, M., and M. Sumbwanyambe. 2019. "An Algorithm for Severity Estimation of Plant Leaf Diseases by the Use of Colour Threshold Image Segmentation and Fuzzy Logic Inference: A Proposed Algorithm to Update a 'Leaf Doctor' Application." AgriEngineering 1:205-219.

Xie, W., K. Yu, K. P. Pauls, and A. Navabi. 2012. "Application of Image Analysis in Studies of Quantitative Disease Resistance, Exemplified Using Common Bacterial BlightCommon Bean Pathosystem." Phytopathology 102:434-442. 